\title{
She Would Be the First Sentence of My Next Novel
}

\author{
Nicole Brossard
}

She would be the first sentence of my next novel. Voilà ce qu'elle avait pensé en commençant le texte de sa conférence.

ll y avait maintenant plus d'un an qu'elle songeait à son prochain roman. Le roman s'organiserait autour de ce qui, maintenant énigmatique en elle, se déploierait dans quelques mois majestueusement comme une longue métaphore de vie ou cruellement au rythme de la conscience qui ne laisserait rien au hasard. Elle aimait cet état précurseur de dimension nouvelle, état qui la rendait vulnérable mais qui toujours s'affirmait comme un signe d'espoir. Signe certain que tout ce qu'elle avait vécu, pensé ou lu allait avoir une suite et que, dans l'espace inédit du prochain roman, elle parviendrait peut-être à percer quelque secret de la condition humaine, resté indéchiffrable jusqu'à ce jour. Pour le moment, il y avait une euphorie sans récit, une myriade d'images qui valaient mille récits, qui voilaient le récit. Chaque fois qu'un élément de récit était sur le point de prendre forme, elle laissait faire un instant la forme, puis si la forme se transformait en un sujet, alors elle notait non pas le sujet mais comment la forme s'était transformée.

Ainsi le sujet de son prochain roman pouvait-il lui échapper pendant des mois obsédant et inaccessible. Sujet qui lui semblait toujours proche et lointain comme l'empreinte visuelle d'un monde à rattraper, enfoui dans le nombre effarant des permutations sémantiques, perdu dans l'immensité de l'espace et de l'espèce, monde infiniment précieux que la conscience allait tenter de réinsérer dans le langage ou d'en imaginer le versant inédit. 